\title{
Spherical nanosized focal spot unravels the interior of cells
}

\author{
Roman Schmidt $^{1}$, Christian A Wurm ${ }^{1}$, Stefan Jakobs ${ }^{1}$, Johann Engelhardt ${ }^{2}$, Alexander Egner ${ }^{1}$ \& Stefan W Hell ${ }^{1,2}$
}

The resolution of any linear imaging system is given by its point spread function (PSF) that quantifies the blur of an object point in the image. The sharper the PSF, the better the resolution is. In standard fluorescence microscopy, however, diffraction dictates a PSF with a cigar-shaped main maximum, called the focal spot, which extends over at least half the wavelength of light $(\lambda=400-700 \mathrm{~nm})$ in the focal plane and $>\lambda$ along the optical axis (z). Although concepts have been developed to sharpen the focal spot both laterally and axially, none of them has reached their ultimate goal: a spherical spot that can be arbitrarily downscaled in size. Here we introduce a fluorescence microscope that creates nearly spherical focal spots of 40-45 nm $(\lambda / 16)$ in diameter. Fully relying on focused light, this lens-based fluorescence nanoscope unravels the interior of cells noninvasively, uniquely dissecting their sub- $\lambda$-sized organelles.

Far-field fluorescence microscopy is exceptional in its ability to noninvasively image the interior of cells with molecular specificity

and in three dimensions. For many decades, however, the resolution of its standard variants have been limited by a main point spread function (PSF) maximum having a cross-sectional diameter of $>200 \mathrm{~nm}$ in the focal plane $(x, y)$ and a length of $>500 \mathrm{~nm}$ along the optic axis $(z)$. To overcome these limits, microscopy concepts have emerged to shrink the size of the effective focal spot. By coherently adding the spherical wavefronts of two opposing lenses, $4 \mathrm{Pi}$ microscopy ${ }^{1-4}$ and $\mathrm{I}^{5} \mathrm{M}$ (ref. 5) have reduced the main maximum of the PSF by a factor of 3-7 along the optic axis. A more fundamental reduction has been attained by exploiting molecular transitions of the fluorophore specifically for this purpose ${ }^{6,7}$. For example, in stimulated emission depletion (STED) microscopy ${ }^{8}$, the main PSF extent is decreased to a small fraction of the wavelength, $\lambda$, by overlapping the excitation light with a light distribution that features a local intensity zero. The excited fluorophores are quenched by the overlapping light distribution except at the zero. Thus the fluorophore excitation is effectively confined to the proximity of the zero, rendering a very narrow PSF. Molecules falling within the PSF maximum can contribute to the signal at the same time. When implemented in a scanning microscope, the subdiffraction-sized PSF yields images with subdiffraction resolution ${ }^{8-10}$.

Notably, any fluorophore process that reversibly inhibits fluorescence generation can be used to break the diffraction barrier ${ }^{6,7}$. Therefore, related schemes have used other intramolecular fluorophore transitions to squeeze the focal spot and hence to sharpen the PSF, such as the depletion of the fluorophore ground state $\mathrm{e}^{11,12}$ or the switching of photochromic molecules between a fluorescence activated 'on' and a deactivated 'off' state ${ }^{13}$. The sharpening of the focal spot by PSF engineering ${ }^{6,7}$ is equivalent to expanding the microscope's spatial frequency pass-band, which, using fluorophore transitions, can also be achieved in highly parallelized recording schemes that narrow the PSF with arrays of zeros ${ }^{14}$.

Molecular switching has also opened the door to powerful superresolution schemes that switch photochromic molecules individually in a widefield illumination microscope, so as to mathematically localize them through the bunch of photons emitted in the 'on' state $^{15-18}$. These concepts use, and in fact rely on, the assumption of switching on a single molecule at a time in the diffraction volume. Although an impressive simultaneous gain in lateral and axial resolution has recently been demonstrated ${ }^{19}$, obtaining such images has so far required fluorophores that are both switchable and exceptionally bright. Moreover, as the object is read out molecule by molecule, this approach has to avoid background signal from the body of the cell. Therefore achieving superresolution by single-molecule switching, as opposed to by PSF engineering ${ }^{6,7}$, has so far been limited to thin object layers that are close to the coverslip ${ }^{19}$. Although the resolution increase provided by single-molecule switching concepts can be extended to the $z$ axis ${ }^{19}$, these concepts inherently lack three-dimensional (3D) optical sectioning, unless they are combined with multiphoton excitation ${ }^{20}$.

Methods based on sharpening of the $\mathrm{PSF}^{6,7}$, in contrast, are attractive for imaging deep inside a cell. First, spot- or multiple spot-oriented schemes are applicable to normal (nonswitchable) fluorophores. Second, because they detect molecular ensembles, they allow the tuning of the signal strength by varying the spot size, which is particularly important for the nanoscale imaging inside living cells ${ }^{21}$. Third, as they are ensemble methods, schemes

\footnotetext{
${ }^{1}$ Max Planck Institute for Biophysical Chemistry, Department of NanoBiophotonics, Am Fassberg 11, 37077 Göttingen, Germany. ${ }^{2}$ German Cancer Research Center (DKFZ), High-Resolution Optical Microscopy Division, Im Neuenheimer Feld 280, 69120 Heidelberg, Germany. Correspondence should be addressed to A.E. (aegner@gwdg.de) or S.W.H. (shell@gwdg.de).
}

RECEIVED 31 MARCH; ACCEPTED 23 APRIL; PUBLISHED ONLINE 18 MAY 2008; DOI:10.1038/NMETH.1214 
Figure 1 | Fluorescence microscopy setup with isotropic 3D focal spot. (a) Beams for excitation, STEDxy (lateral) and STEDz (axial) are combined using a dichroic mirror (DCSP) and then fed through a beam scanner into a 4Pi unit with two opposing objective lenses ( 01 and $02 ; \mathrm{HCX}$ PL APO $100 \times, 1.46$ NA OIL CORR; Leica Microsystems). The fluorescence light (orange) collected by both lenses backpropagates along the same optical path to the detector, passing through the DCSP and a dichroic mirror (DCLP). The pivot plane (PP) of all scanning beams is conjugated to the entrance pupils of the objective lenses. The incoming beams are divided by a polarizing beam-splitter (PBS) and coherently superimposed at both lenses' common focal plane inside the sample (S). A piezodriven mirror (MP) controls the difference in pathlength between both cavity arms and thereby the 4Pi phases of all beams. The polarization state of STEDxy and STEDz is adjusted by two half-wave retarder plates ( $\mathrm{H} 1$ and $\mathrm{H} 2)$. Numbers in black circles indicate points for which these polarization states are displayed in c. *, see Figure 2 for focal intensity distributions. (b) The excitation beam and the STED beams for lateral (STEDxy, imprinted with a circular phase ramp (PM)) and axial (STEDz) fluorescent spot compression are polarized under $\alpha=45^{\circ}$ with respect to the perpendicular direction $(n)$ to the splitting plane $(p)$ of the polarizing beam-splitter. STEDxy and STEDz are polarized orthogonal to each other. (c) Polarization state of STEDxy (outlined) and STEDz (solid) at indicated points in the setup. By tuning the orientation of the half wave retarder plate $\mathrm{H} 1$ and thereby $\alpha$, the branching ratio between both arms is adjusted for matching intensities of the partial beams (position 1 and 2). Right behind the polarizing beam-splitter, the partial beams of STEDxy and STEDz are mutually orthogonally polarized (positions 3 and $3^{\prime}$ ). Parallel polarization for both beam pairs is rectified by the second half wave retarder plate $\mathrm{H} 2$ (positions 4 and $4^{\prime}$ ). Notably, the relative phase between the STEDxy and STEDz beam pairs now differs by $\pi$. 2elying on PSF sharpening can, in conjunction with confocal detection, effectively suppress the background, which renders them single molecule-sensitive in the bulk. Directing the focal spot to an arbitrary coordinate in the sample allows direct probing of sites of interest in the cellular interior. However, to unfold its full 3D imaging potential, the PSF must be spherical; in fact, one can argue that the $3 \mathrm{D}$ resolution is limited by its weakest part (Supplementary Note online). Unfortunately, owing to the highly asymmetric convergence of the focused wavefront at the focal point of a lens, isotropic focal spots are not readily attained.

We have now solved this problem by encircling the focal point of a scanning fluorescence microscope by a focal intensity distribution, depleting potentially excited molecules by stimulated emission. The net result was an effective PSF delivering a virtually isotropic spatial resolution of $40-45 \mathrm{~nm}(\lambda / 16)$. Threedimensional nanoscale imaging of mitochondria, in morphologically intact fixed mammalian cells, revealed that although the mitochondrial protein Tom 20 clearly delineated the mitochondrial outer membrane, Tom 20 was predominantly assembled in clusters. We finally showed that isotropic 3D nanoscopy can be extended to multicolor imaging by adding an additional color channel.

\section{RESULTS}

\section{Spherical focal spot generation}

To generate a light field that enables isotropic compression of the fluorescent spot, we used two opposing lenses to allow the coherent addition of the wavefronts in the focal region. Contrary to a standard 4Pi microscope $\mathrm{P}^{1-4}$, we used a polarizing beam-splitter for producing the counter-propagating beam pairs. To still allow coherent field addition in the focal region, we rectified the polarization in one of the arms using a $\lambda / 2$ retarder (Fig. 1a). The use of a polarizing beam-splitter was important in this arrangement because it allowed us to match the amplitudes of the two outgoing partial beams by tilting the polarization plane of an incident beam by an angle $\alpha$ of about $45^{\circ}$ with respect to that of the beam-splitter (Fig. 1b). Moreover, it allowed us to synthesize the STED intensity patterns by the (incoherent) addition of two differently but simultaneously generated light patterns featuring a common focal zero: one for lateral (STEDxy) and one for axial (STEDz) squeezing ${ }^{10}$.

To prevent these patterns from interfering with each other, we produced them at the same wavelength, $\lambda_{\mathrm{STED}}$, but with two different lasers. We tilted the polarization of the two incoherent laser beams by $45^{\circ}$ with respect to that of the splitter but kept them orthogonally oriented with respect to each other (Fig. 1b,c). Before the wavefront of the STEDxy beam entered the triangular cavity, we imprinted it with a circular phase ramp to render a doughnut shape in the focal region. Constructive interference of the phase-modified partial beams at the focal point doubled the field intensity in the doughnut, which led to a more efficient depletion compared to a single-lens setup, while the central zero was left intact. The regularly focused partial waves of the STEDz beam also interfered at the focal point, but owing to its orthogonal polarization orientation with respect to its STEDxy counterpart (Fig. 1c), the partial beams of the STEDz beam interfered destructively, which rendered a central zero with steep edges along the $z$ axis. Thus, the polarizing beam-splitter tightly locked the two patterns to 'orthogonal' interference. The superposition of these focal intensities yielded a focal '3D doughnut' of uniform polarization state that virtually enclosed the geometrical focal point (Fig. 2).

We advantageously selected an optical path length difference that, besides installing the above pattern for fluorescence inhibition, rendered constructive interference for the excitation light at the excitation wavelength, $\lambda_{\mathrm{e}}$, provided by the laser. Whereas the axial extent of the main maximum of the PSF was thus reduced by a factor of 3, the actual benefit here was that the excitation focal volume was cut down by half, which reduced the photobleaching by the same factor. Making STEDxy about fivefold more intense than STEDz ensured that the intensity profiles, $I_{\text {STED }}$ (Fig. 2e), around the common central zero intensity point were equally steep along $x$, $y$ and $z$ axes. As a result, the fluorescence spot was isotropically compressed following a square-root $\operatorname{law}^{22}$ to a subdiffraction-sized 

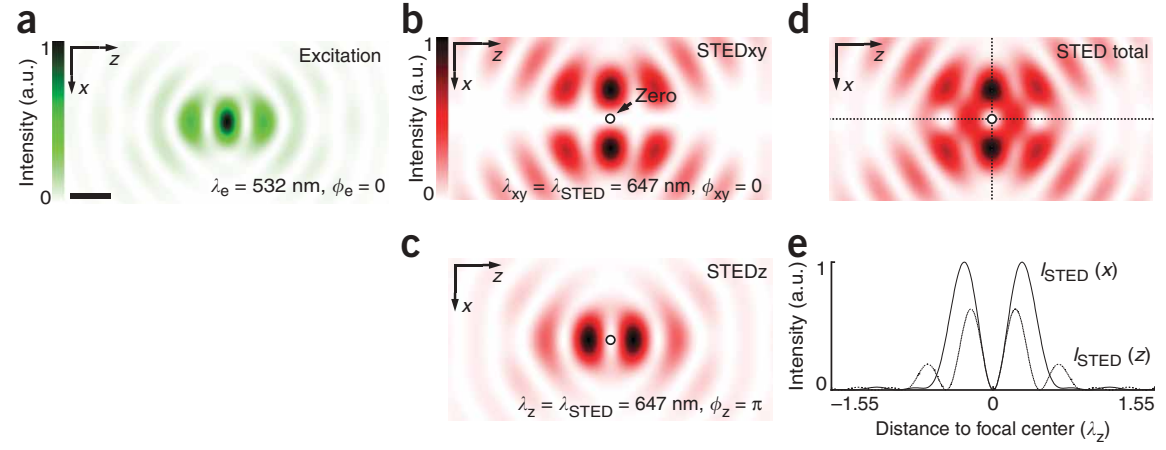

Figure 2 | Calculated focal intensity distributions and formation of the STED PSF with respective wavelengths, $\lambda$, and $4 \mathrm{Pi}$ phases, $\phi$. (a) Constructive $\left(\phi_{\mathrm{e}}=0\right)$ interference pattern of the excitation light. (b) Constructive $\left(\phi_{x y}=0\right)$ interference pattern of STEDxy. The doughnut-like shape is generated by a phase mask (PM; Fig. 1a). (c) Destructive $\left(\phi_{z}=\pi\right)$ interference pattern of STEDz. (d) Incoherent superposition of both STED beams with relative focused power of 1.0 (STEDxy) and 0.2 (STEDz). (e) Focal STED intensity profiles along $x$ and $z$ axes (d, dotted lines). The matching intensity gradients enable isotropic compression of the fluorescent spot and hence of the effective PSF of the microscope. Circles mark central points of vanishing field intensities. All calculations were carried out for a $1.4 \mathrm{NA}$ oil immersion lens. Scale bar, $250 \mathrm{~nm}$.

sphere of diameter $d$ :

$$
d \approx \lambda /\left(2 \mathrm{NA} \sqrt{1+I_{\mathrm{m}} / I_{\mathrm{s}}}\right)
$$

with NA denoting the numerical aperture of the lens, $I_{\mathrm{m}}=$ maximum $\left(I_{\mathrm{STED}}(z)\right)$, and $I_{\mathrm{S}}$ denoting the intensity at which the ability of the fluorophore to emit is cut down by half. We termed this concept isoSTED, because for $I_{\mathrm{m}}>5 I_{\mathrm{s}}$, the fluorescence spot became essentially a sphere with a diameter

$$
d \approx \lambda /\left(n \pi \sqrt{I_{\mathrm{m}} / I_{\mathrm{s}}}\right),
$$

where $n$ is the refractive index of the sample. For $I_{\mathrm{m}} / I_{\mathrm{s}} \rightarrow \infty$ and a perfect central zero, the spherical fluorescence spot uniformly converges to a point.

\section{Experimental verification and application}

Based on the principle described above, we built the isoSTED microscope. We compared the calculated effective PSF of a confocal fluorescence microscope at $\mathrm{NA}=1.4$, $\lambda_{\mathrm{e}}=532 \mathrm{~nm}$ and fluorescence wavelength $\lambda_{\mathrm{f}}=580 \mathrm{~nm}$ with that of an isoSTED microscope using the same lenses and operating with $\lambda_{\mathrm{STED}}=647 \mathrm{~nm}$ and $I_{\mathrm{m}} / I_{\mathrm{s}}=15$ (Fig. 3a). These parameters actually corresponded to our experimental implementation, which used two actively mode-locked and synchronized ion lasers delivering $\sim 200$-ps pulses at $72-\mathrm{MHz}$ repetition rate. The excitation pulses of $<100$ ps duration were provided by a laser diode that was synchronized to the mode-locker of the ion lasers. We verified the isotropic PSF of our system by imaging 21-nm-diameter fluorescent beads (Fig. 3b). In the normal confocal mode (that is, illumination through a single lens and no STED beam, $\mathrm{NA}=1.46)$, the full-width-half-maximum

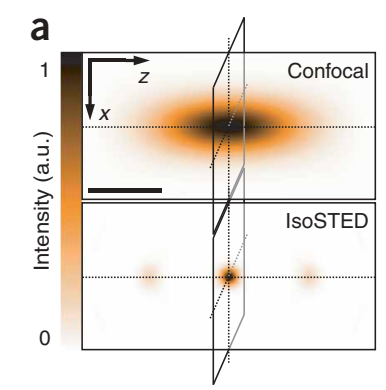
are marked with colored circles. Scale bars, $250 \mathrm{~nm}$.
(FWHM) of the focal spot amounted to 165 $\mathrm{nm}$ in the lateral and $405 \mathrm{~nm}$ in the axial direction. Switching on the isoSTED mode with an average power of (merely) $P_{\mathrm{xy}}=20 \mathrm{~mW}$ for the lateral and $P_{\mathrm{z}}=3 \mathrm{~mW}$ for the axial STED beam, confined the fluorescence generation mainly to the central zero of the focal STED pattern. In good agreement with the calculation, we obtained a nearly spherical main maximum of the PSF displaying a FWHM of 40, 41 and $44 \mathrm{~nm}$ in the $x, y$ and $z$ directions, respectively (Fig. 3c). The only difference to the calculation was that some signal $(<35 \%$ of the main peak) was also generated at the first-order side-minima along the optical axis, a feature of the PSF that can be readily dealt with by linear or nonlinear deconvolution of the image data. Notwithstanding this, the experimental isoSTED PSF was dominated by a main maximum delivering an isotropic 3D resolution of $\sim \lambda / 16$.

Next we investigated the distributions of mitochondrial proteins in morphologically intact fixed mammalian cells. Mitochondria are spherical- to tubular-shaped organelles with inner and outer membranes. So far, the 200-400 nm diameter of this organelle has virtually prevented the visualization of protein spatial distributions in mitochondria by light microscopy. The translocase of the mitochondrial outer membrane (TOM complex) serves as the mitochondrial entry gate for the vast majority of nuclear-encoded protein precursors ${ }^{23-25}$. Acting as a primary import receptor, Tom 20 is a subunit of the TOM complex. Biochemical evidence suggests that the TOM complex is enriched in so-called contact-sites involved in the translocation of proteins. We immunologically labeled Tom 20 with the orange-emitting organic fluorophore NK51 (Atto-Tec). We chose this fluorophore because the laser lines for $\lambda_{\mathrm{e}}$ and $\lambda_{\text {STED }}$ perfectly match its excitation and emission spectra.

Three $<50 \mathrm{~nm}$ thin optical sections ( $x-y$ images) through a mitochondrion inside a mammalian (Vero) cell after nonlinear deconvolution, recorded at the bottom, center and the top of the
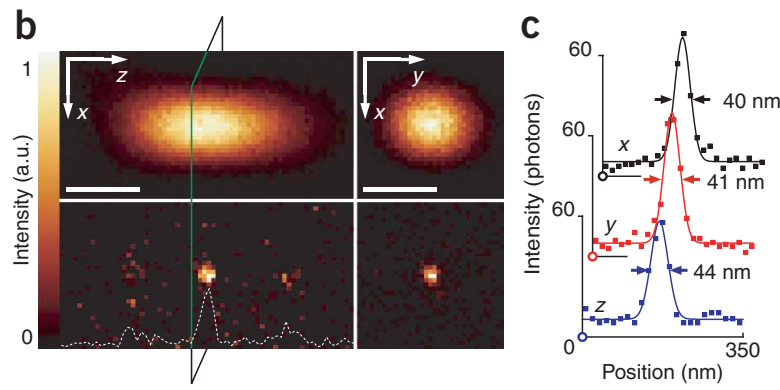

Figure 3 | Isotropic effective focal spot (PSF) on the nanoscale. (a) Calculated PSF of a confocal fluorescence microscope and the corresponding spherical PSF of the isOSTED microscope at $I_{\mathrm{m}} / I_{\mathrm{s}}=15$ (NA = 1.4; wavelength parameters as in Fig. 2). (b) Experimental counterpart to a as measured with a 21-nm-diameter fluorescence bead. The FWHM of the confocal setup (1.46 NA) is $165 \mathrm{~nm}$ in the lateral and $405 \mathrm{~nm}$ in the axial direction. Switching on the STED pattern shown in Figure $\mathbf{2 d}$ leads to a largely spherical main focal fluorescence spot. (c) Gaussian fits through the lateral and axial profiles of the focal spot yield indicated FWHM, corresponding to an isotropic far-field optical 3D resolution of $\lambda / 16$. Baselines 

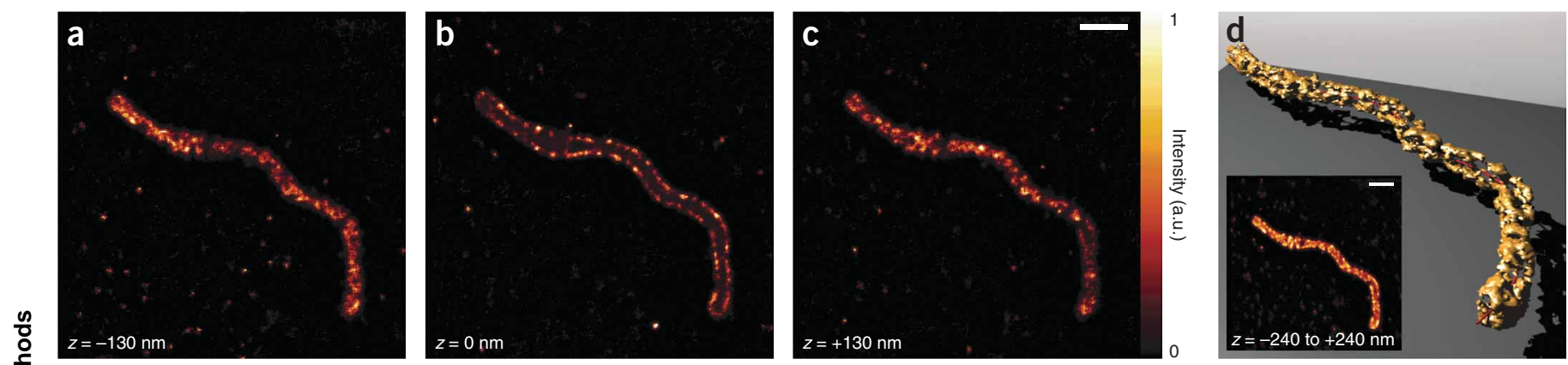

Figure 4 | IsOSTED fluorescence microscopy dissects a mitochondrion by focusing light into the interior of a mammalian (Vero) cell; here the distribution of the protein Tom20 is imaged. (a-c) Optical sections through the bottom (a), center (b) and top (c) planes of the mitochondrion reveal distinct Tom20 clusters. The $x-y$ image through the center plane shows that the clusters are located at the rim of the organelle. The sections shown are part of a 3D stack of $62 x-y$ images, all equally spaced in the $z$ dimension by $22 \mathrm{~nm}$. The image data shown were obtained after deconvolution as described in Methods. (d) 3D reconstruction of the Tom20 distribution (the red filament is to guide the eye). Inset, sum of all $x-y$ images from $z=-240 \mathrm{~nm}$ to $z=+240 \mathrm{~nm}$. This mimics a nanoresolving (STED) microscope with standard confocal resolution along the $z$ direction, showing that axial super-resolution is essential for unraveling the protein distribution in this sub-wavelength-sized organelle. Scale bars, $1 \mu \mathrm{m}$.

organelle, revealed that Tom20 forms distinct clusters at the organelle's boundary (Fig. $\mathbf{4 a - c}$ ). Since the $x-y$ images are part of a 3D stack of $62 x-y$ images (equally spaced in the $z$ dimension by

$22 \mathrm{~nm}$ ), we rendered a 3D representation of the Tom20 distribution (Fig. 4d). The isotropic resolution was essential to identify the peripheral localization of Tom20. Summing up all $x-y$ images simulated a purely two-dimensional nanoscopic method. The resulting image (Fig. 4d) revealed that a method lacking axial subdiffraction resolution cannot be used to identify the location of the clusters at the periphery of the organelle.

\section{Dual-color 3D nanoscopy imaging}

To demonstrate the ability of isoSTED to image the spatial relationship of two or more biomolecules at the nanoscale in three dimensions, we added a second color channel. The organic fluorophore DY-485XL (Dyomics GmbH) exhibits a similar emission spectrum as NK51 and hence can be quenched by the same $\lambda_{\text {STED }}=$ $647 \mathrm{~nm}$. However, as its excitation spectrum is blue-shifted by $\sim 50 \mathrm{~nm}$ (Fig. 5), we could discriminate between these fluorophores by choosing $488 \mathrm{~nm}$ as the excitation wavelength for color channel 1 (DY-485XL) and the customary $532 \mathrm{~nm}$ for channel 2 (NK51). This scheme was very attractive because it used the same STED beam zero for both channels, which therefore were inherently aligned with respect to each other. We removed the residual excitation cross-talk between the two channels by linear unmixing of the raw data.

As a second protein we investigated the matrix protein $\mathrm{mtHsp} 70$ (also referred to as mortalin or GRP 75), a component of the mitochondrial protein import motor ${ }^{23-25}$. Regulated cycles of $\mathrm{mtHsp} 70$ binding to precursor proteins and its subsequent release

Figure 5 | Two-color isoSTED imaging of mitochondria in Vero cells.

$(\mathbf{a}, \mathbf{b})$ Distribution of the outer membrane protein Tom20 labeled with the organic fluorophore DY-485XL (a) and of the NK51-labeled matrix protein $\mathrm{mtHsp70}$ (b). (c) Overlay of the two images showing Tom20 in green and $\mathrm{mtHsp70}$ in red. (d) Excitation and absorption spectra for DY-485XL (dotted line) and NK51 (solid line). The two fluorophores are separated by subsequently exciting the sample at $488 \mathrm{~nm}$ (DY-485XL) and $532 \mathrm{~nm}$ (NK51). Both dyes are depleted by stimulated emission at $647 \mathrm{~nm}$. (e) Reverse staining as a control (Tom20 with NK51 and Hsp70 with DY-485XL) yields similar results as the initial recording (c). Scale bars, $1 \mu \mathrm{m}$.

mediate vectorial movement of the precursor proteins toward the matrix. The intermittent association of $\mathrm{mtHsp70}$ with the import machinery may suggest that a sizeable fraction of the $\mathrm{mtHsp} 70$ pool is at any time associated with the inner membrane of the mitochondrion. However, we did not find any enrichment of mtHsp70 at the mitochondrial rim, indicating that the majority of mtHsp70 is located in the mitochondrial matrix (Fig. 5b).

The features visible in the individual images of Tom20 (DY485XL; Fig. 5a) and mtHsp70 (NK51; Fig. 5b) in the middle plane of the very same mitochondrion are equivalent to those observed in single-color experiments (Fig. 4) and therefore indicate that the isotropic resolution is in the 50-nm range for both channels. The details in channel 2 (NK51) appear to be slightly sharper, which is due to a comparatively better STED efficiency of this dye at $647 \mathrm{~nm}$. As a control, we reversed the labeling of the two proteins and obtained essentially the same features (Fig. 5e). In both cases, the Tom 20 protein clearly delineated the mitochondrion. Note that apart from the linear unmixing, the shown data (Fig. 5) are raw, that is, non-deconvolved. The dual-color images (Fig. $\mathbf{5 c}, \mathbf{e}$ ) are, to
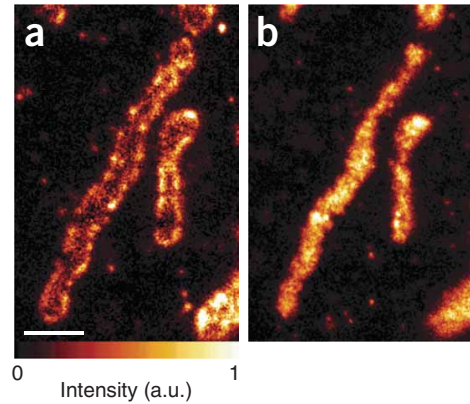

Intensity (a.u.)

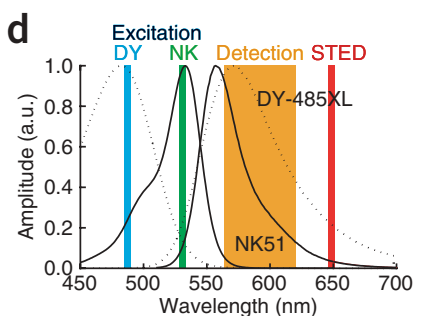

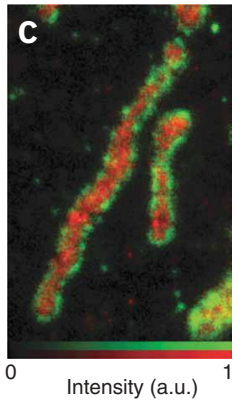

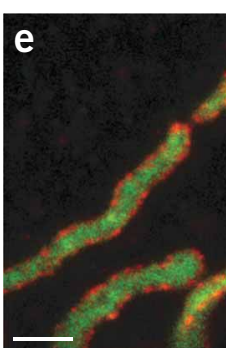


our knowledge, the first light microscopy recordings separating two proteins of an organelle inside a whole cell with nanoscale 3D resolution, demonstrating the emerging capability of optical microscopy to image the interior of nanosized subcellular organelles with regular lenses and visible light.

\section{DISCUSSION}

An important advantage of our 4Pi-like arrangement is that, for symmetry reasons, it inherently cancels the focal fields of all the STED beams in the geometric focal point, regardless of their polarization. This attribute facilitated the conceptually flawless implementation of a 3D zero. Therefore, the optical scheme can readily be modified for circular polarization by inserting mutually orthogonal quarter-wave retarders in each cavity arm so that the partial beams are orthogonal in their circular polarization. Circularly polarized light advantageously addresses the molecules irrespective of their orientation in the focal plane.

Instead of polarization, one could have also resorted to wavelength differences for forming the STEDxy and STEDz focal patterns. However, such a scheme would have made it challenging to identify the specific cavity path difference that reconciles the formation of destructive interference for STEDz, constructive interference for STEDxy and constructive interference for the excitation light. In contrast, operating with the same $\lambda_{\text {STED }}$ and a polarizing beamsplitter inherently provides the required relative phase difference.

In the future it will also be possible to resort to pulsed laser systems with noncoherent sequential pulses, making the use of two STED lasers obsolete. Further, photonic crystal devices and continuous wave lasers will greatly simplify future implementations of isoSTED microscopy. In any case, the scheme presented in this work (Fig. 1) and its derivatives are rather definitive with regard to the general requirements for an optical system to compress a focal fluorescence spot by light. This is important because stimulated emission is just one way of reversibly inhibiting fluorescence. Other known mechanisms include the depletion of the fluorescence ground state ${ }^{11,12}$ and photoswitching of molecules between a fluorescent and a nonfluorescent state ${ }^{13}$. In all these cases, the resolution will follow the above equation determined by $I_{\mathrm{m}} / I_{\mathrm{s}}$, with $I_{\mathrm{s}}$ denoting the saturation intensity for the particular molecular mechanism. The latter can be lower than the values used for STED by several orders of magnitude. Although it has not been exploited in this study, a major advantage of a far-field optical method working with molecular ensembles is that the focal spot volume can be tuned in size by adjusting $I_{\mathrm{m}} / I_{\mathrm{s}}$. This feature should be highly advantageous when translating this concept to 3D imaging inside living cells.

Further improvements should yield isotropic PSFs that are substantially smaller than the $\sim 40 \mathrm{~nm}$ reported in this study. It should be feasible to generate isotropic focal spots whose diameter can be continuously tuned in the nanometer range to accommodate the specific signal and resolution needs of the object to be investigated. The fact that our isoSTED data were obtained by implementing the $4 \mathrm{Pi}$-cavity in a standard beam scanning confocal microscope (Leica TCS NT) with fast galvanometric beam deflectors underscores the potential of our scheme for future applications in the life sciences and beyond.

\section{METHODS}

Laser sources and timing. We used a pulsed STED scheme in which a short excitation pulse was immediately followed by two simultaneous STED pulses (STEDxy for lateral and STEDz for axial confinement). We linked four laser systems in a master-slave relationship and operated them at a pulse-repetition rate of $72 \mathrm{MHz}$. A mode-locked krypton-ion laser (modified Innova Sabre; Coherent Inc.) served as master and provided the STEDxy pulse. The STEDz pulse originated from a second mode-locked mixed gas argon/krypton-ion laser (modified Innova 70C Spectrum; Coherent Inc.). The same HF oscillator (custom-built PulseDrive Dual; APE) drove the mode-locker units of both lasers, with a variable phase between them, thus generating two synchronized pulse trains with an adjustable time delay. The original width of both pulses was $200 \mathrm{ps}$, which was still a factor of ten smaller than the lifetime of a typical fluorophore ( $\sim 2 \mathrm{~ns})$, but already long enough to prevent damage to the sample by nonlinear processes. Unlike with femtosecond mode-locked titanium: sapphire lasers and optical parametric oscillator systems, we therefore eliminated the need for pulse stretching. We coupled out a small fraction of the STEDz pulse to a fast photodiode that triggered one of the two alternative excitation semiconductor lasers (PicoTA 530 and PicoTA 490, pulse width < 100 ps; PicoQuant) via a custom-built electronic picosecond-delay unit. We performed the temporal alignment by measuring the received fluorescence from a remote part of the sample and adjusted all delays individually for maximum STED efficiency.

Image deconvolution. We applied a maximum likelihood expectation maximization ${ }^{26}$ method to remove the effect of the side lobes above and below the main spherical focal spot in the 3D image stack (Fig. 4). We obtained a first estimate of the PSF from a small subset of the raw data. Then we deconvolved the whole dataset by 15 iterations of a Richardson-Lucy algorithm ${ }^{27}$. We then assessed the quality of the PSF estimate by measuring the spurious signal outside the organelle. We obtained the final data by iterative refinement of the PSF estimate with regard to the minimization of this signal.

Cross-talk determination and spectral unmixing. In the two color experiments (Fig. 5), we used DY-485XL and NK51 as fluorophores. Both exhibit almost similar emission spectra and therefore could be both depleted by STED light of the same wavelength. To distinguish between the two dyes, we excited the sample consecutively with $488 \mathrm{~nm}$ (DY-485XL, channel 1) and $532 \mathrm{~nm}$ (NK51, channel 2) radiation. Although the excitation wavelengths were separated by more than $40 \mathrm{~nm}$ and corresponded well to the respective dye's absorption maximum, the other fluorophore was also slightly excited. This introduced some cross-talk in our data, resulting in pixel counts of channel 1 and channel 2 that were a linear combination of the contributions from both dyes. As the cross-talk could be determined, we used linear spectral unmixing ${ }^{28}$ to separate the DY-485XL and NK51 fluorescence. The strength of the cross-talk depended on the relative intensities of the excitation lasers and therefore varied slightly for each recording. Therefore, we considered only those sets of images for further analysis that contained enough information to directly determine the unmixing parameters. Most of our recordings contained isolated agglomerations of secondary antibodies. Plotting all pixel values (corresponding to isolated agglomerations) in channel 1-channel 2 space showed that two distinct and well-defined groups could be identified, representing agglomerations consisting of either DY-485XL-conjugated or NK51-conjugated antibodies. We 
quantified the magnitude of the cross-talk by analyzing both groups. We determined that for the data shown in Figure 5a-c, the ratio between channel 1 and channel 2 was 84:16 for DY-485XL and 23:77 for NK51. The corresponding values for the control (Fig. 5e) were 86:14 and 25:75.

Sample preparation. For PSF measurements, we placed $10 \mu \mathrm{l}$ of a 1:10,000 diluted suspension of fluorescent beads (Nile red; specified diameter, $21 \mathrm{~nm}$; $2 \%$ solids in distilled water; Molecular Probes) on a coverslip. After drying, we covered the sample with a thin layer of $97 \%$ 2,2'-thiodiethanol (TDE) in phosphate-buffered saline (PBS; $137 \mathrm{mM} \mathrm{NaCl}, 3 \mathrm{mM} \mathrm{KCl}, 8 \mathrm{mM} \mathrm{Na}_{2} \mathrm{HPO}_{4}, 1.5 \mathrm{mM}$ $\mathrm{KH}_{2} \mathrm{PO}_{4} ; \mathrm{pH} 7$ ) (refractive index $n=1.515$ at room temperature $)^{29}$ and a second coverslip. We finally sealed the sample with nail polish and mounted it in the isoSTED microscope.

For immunological labeling, we grew cultured Vero cells from the African green monkey, Chlorocebus sp., on coverslips. We fixed the cells with $8 \%$ formaldehyde in PBS for $3-10 \mathrm{~min}$ at $37{ }^{\circ} \mathrm{C}$, extracted them with $0.5 \%$ (vol/vol) Triton X-100 in PBS, blocked with 5\% BSA in PBS and incubated them with a polyclonal rabbit antiserum to Tom20 (Santa Cruz Biotechnology) or with mouse monoclonal antibodies to mtHsp70 (Abcam). We detected the primary antibodies with secondary antibodies (sheep anti-mouse, goat anti-rabbit) that were custom-labeled with NK51 (AttoTec) or DY-485XL (Dyomics GmbH). For single-color recording (Fig. 4), we used custom-labeled tertiary antibodies (donkey anti-goat; Jackson ImmunoResearch Laboratories) to intensify the brightness of the detected structures. After labeling, we exchanged the buffer by a dilution series of TDE in PBS as described $^{29}$, finally resulting in an embedding medium of $97 \%$ TDE in PBS $(n=1.515)$. This embedding strategy greatly improves the optical properties of the sample and therefore prevents that local refractive index changes might hamper resolution $^{29}$. We covered the samples with a second coverslip that was sparsely coated with fluorescent beads (Nile red; specified diameter, $110 \mathrm{~nm}$; Spherotec Inc.) to facilitate the initial alignment of the microscope. We sealed the samples with nail polish and mounted them in the isoSTED microscope.

Note: Supplementary information is available on the Nature Methods website.

\section{ACKNOWLEDGMENTS}

We thank S. Stoldt for help with cell culture. We acknowledge A. Schönle and J. Keller (Max Planck Institute for Biophysical Chemistry) for providing us with the analysis software (Imspector), the PSF calculation software and for helpful discussions. We also acknowledge helpful discussions with $\mathrm{K}$. Willig and thank J. Jethwa for critically reading the manuscript. This work was supported by grants of the Deutsche Forschungsgemeinschaft (JA 1129/3) to S.J. and (SFB 755) to A.E. and S.W.H.

\section{Published online at http://www.nature.com/naturemethods/ Reprints and permissions information is available online at http://npg.nature.com/reprintsandpermissions/}

1. Hell, S.W. Double confocal microscope. European patent 0491289 (1992).

2. Hell, S.W. \& Stelzer, E.H.K. Fundamental improvement of resolution with a $4 \mathrm{Pi}$ confocal fluorescence microscope using two-photon excitation. Opt. Commun. 93, 277-282 (1992).
3. Egner, A., Jakobs, S. \& Hell, S.W. Fast 100-nm resolution 3D-microscope reveals structural plasticity of mitochondria in live yeast. Proc. Natl. Acad. Sci. USA 99, 3370-3375 (2002).

4. Gugel, H. et al. Cooperative 4Pi excitation and detection yields 7-fold sharper optical sections in live cell microscopy. Biophys. J. 87, 4146-4152 (2004).

5. Gustafsson, M.G.L., Agard, D.A. \& Sedat, J.W. I5M: 3D widefield light microscopy with better than $100 \mathrm{~nm}$ axial resolution. J. Microsc. 195, 10-16 (1999).

6. Hell, S.W. Improvement of lateral resolution in far-field light microscopy using two-photon excitation with offset beams. Opt. Commun. 106, 19-24 (1994).

7. Hell, S.W. in Topics in Fluorescence Spectroscopy (ed., Lakowicz, J. R.) 361-422 (Plenum Press, New York, 1997).

8. Hell, S.W. \& Wichmann, J. Breaking the diffraction resolution limit by stimulated emission: stimulated emission depletion microscopy. Opt. Lett. 19, 780-782 (1994).

9. Klar, T.A., Jakobs, S., Dyba, M., Egner, A. \& Hell, S.W. Fluorescence microscopy with diffraction resolution limit broken by stimulated emission. Proc. Natl. Acad. Sci. USA 97, 8206-8210 (2000).

10. Dyba, M. \& Hell, S.W. Focal spots of size $\lambda / 23$ open up far-field fluorescence microscopy at $33 \mathrm{~nm}$ axial resolution. Phys. Rev. Lett. 88, 163901 (2002).

11. Hell, S.W. \& Kroug, M. Ground-state depletion fluorescence microscopy, a concept for breaking the diffraction resolution limit. Appl. Phys. B 60, 495-497 (1995).

12. Bretschneider, S., Eggeling, C. \& Hell, S.W. Breaking the diffraction barrier in fluorescence microscopy by optical shelving. Phys. Rev. Lett. 98, 218103 (2007).

13. Hell, S.W., Jakobs, S. \& Kastrup, L. Imaging and writing at the nanoscale with focused visible light through saturable optical transitions. Appl. Phys. A 77, 859-860 (2003).

14. Gustafsson, M.G.L. Nonlinear structured-illumination microscopy: wide-field fluorescence imaging with theoretically unlimited resolution. Proc. Natl. Acad. Sci. USA 102, 13081-13086 (2005).

15. Rust, M.J., Bates, M. \& Zhuang, X. Sub-diffraction-limit imaging by stochastic optical reconstruction microscopy (STORM). Nat. Methods 3, 793-796 (2006).

16. Betzig, E. et al. Imaging intracellular fluorescent proteins at nanometer resolution. Science 313, 1642-1645 (2006).

17. Hess, S.T., Girirajan, T.P.K. \& Mason, M.D. Ultra-high resolution imaging by fluorescence photoactivation localization microscopy. Biophys. J. 91, 4258-4272 (2006).

18. Egner, A. et al. Fluorescence nanoscopy in whole cells by asnychronous localization of photoswitching emitters. Biophys. J. 93, 3285-3290 (2007).

19. Huang, B., Wang, W., Bates, M. \& Zhuang, X. Three-dimensional super-resolution imaging by stochastic optical reconstruction microscopy. Science $\mathbf{3 1 9}, \mathbf{8 1 0 - 8 1 3}$ (2008).

20. Fölling, J. et al. Photochromic rhodamines provide nanoscopy with optical sectioning. Angew. Chem. Int. Ed. 46, 6266-6270 (2007).

21. Westphal, V. et al. Video-rate far-field optical nanoscopy dissects synaptic vesicle movement. Science 320, 246-249 (2008).

22. Hell, S.W. Strategy for far-field optical imaging and writing without diffraction limit. Phys. Lett. A 326, 140-145 (2004).

23. Hoogenraad, N.J., Ward, L.A. \& Ryan, M.T. Import and assembly of proteins into mitochondria of mammalian cells. Biochim. Biophys. Acta 1592, 97-105 (2002).

24. Rehling, P., Brandner, K. \& Pfanner, N. Mitochondrial import and the twin-pore translocase. Nat. Rev. Mol. Cell Biol. 5, 519-530 (2004).

25. Neupert, W. \& Herrmann, J.M. Translocation of proteins into mitochondria. Annu. Rev. Biochem. 76, 723-749 (2007).

26. Dempster, A.P., Laird, N.M. \& Rubin, D.B. Maximum likelihood from incomplete data via the EM algorithm. J. Roy. Stat. Soc. B 39, 1-38 (1977).

27. Richardson, W.H. Bayesian-based iterative method of image restoration. J. Opt. Soc. Am. 62, 55-59 (1972).

28. Tsurui, H. et al. Seven-color fluorescence imaging of tissue samples based on Fourier spectroscopy and singular value decomposition. J. Histochem. Cytochem. 48, 653-662 (2000).

29. Staudt, T., Lang, M., Medda, R., Engelhardt, J. \& Hell, S.W. 2,2'-Thiodiethanol: a new water soluble mounting medium for high resolution optical microscopy. Microsc. Res. Tech. 70, 1-9 (2007). 